\title{
Meningococcal disease: Facts versus fiction
}

I N 1991, SEVERAL CLUSTERS OF GROUP C MENINGOCOCCAL disease occurred in Canada. Extensive media coverage resulted in widespread public hysteria, even though the clusters were small and restricted in location. This statement from the Infectious Diseases and Immunization Committee of the Canadian Pediatric Society will review the epidemiology of meningococcal disease in Canada in order to put the issue into proper perspective.

Since 1970 the mean number of meningococcal cases reported annually in Canada has been 258 (range 174 to 426 ). During the late 1970 s to mid-1980s, most cases were caused by serogroup B (serotype 2 b). Since 1986, an increasing proportion of cases have been caused by serogroup C (serotype $2 \mathrm{a}$ ), reaching over $60 \%$ in 1990. Such changes could result in a lower level of herd immunity against the new strains and more frequent disease. Although most cases of meningococcal disease occur in infants and children less than 10 years of age, the incidence of disease in adolescents 15 to 19 years usually is slightly higher compared with children 10 to 14 years and young adults.

Vaccines are available for groups A, C, Y and W135. The group A vaccine has been shown to prevent disease in infants, children and adults, and has been very effective in terminating epidemics. Although both the group Y and W135 vaccines are immunogenic, their protective efficacy is unknown. The group $\mathrm{C}$ vaccine is not as effective in preventing diseases in infants less than two years compared with older children and adults (among whom the efficacy is at least 90\%). However, because infants are at greatest risk of disease and because many do achieve protective anti- $\mathrm{C}$ antibody levels following vaccination, use of the vaccine should be considered in outbreak control in children six months to 19 years.

The situation which occurred in Ontario and Quebec in 1991 was very unusual. Several small clusters of group $\mathrm{C}$ disease had been reported in Quebec earlier that year. In early December, a cluster occurred in the Ottawa-Hull region with a high case-fatality rate. Because of the clusters of cases in St Jerome and other regions in Quebec, and because of the additional cases - three of which were fatal - which occurred in OttawaHull in early January 1992, a decision was made by public health authorities in Ontario and Quebec to undertake a mass immunization campaign in areas where clusters occurred. There were several factors leading to this decision: a disproportionately high number of cases among adolescents; the disproportionately high rate of fulminant meningococcemia leading to a high case-fatality rate; previous examples of clusters of cases in adolescents being followed by more widespread outbreaks in young children in the community; and the occurrence of the outbreak in December/January, prior to the usual seasonal peak of meningococcal in February/March, and the concern that the situation would worsen.

The immunization programs were completed by early February. No additional clusters of cases have been observed in areas in which immunization was carried out.

Meningococcal vaccine is not recommended for routine use in Canada because: disease frequently is caused by serogroup B strains for which there is no effective vaccine; the available vaccines are not very effective in infants less than 24 months, the group at greatest risk of disease; and even in older children and adults, protection is not long-lasting, necessitating frequent boosters.

The use of meningococcal vaccine in Canada is recommended only when increased disease incidence or clusters of cases are observed. Detailed recommendations relating to the control of meningococcal disease have recently been published by the Advisory Committee on Epidemiology and should be consulted (1).

\section{REFERENCES}

1. Advisory Committee on Epidemiology. Guidelines for control of meningococcal disease. Can Dis Weekly Rep 1991:17:245-50. 


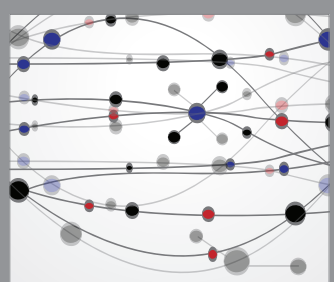

The Scientific World Journal
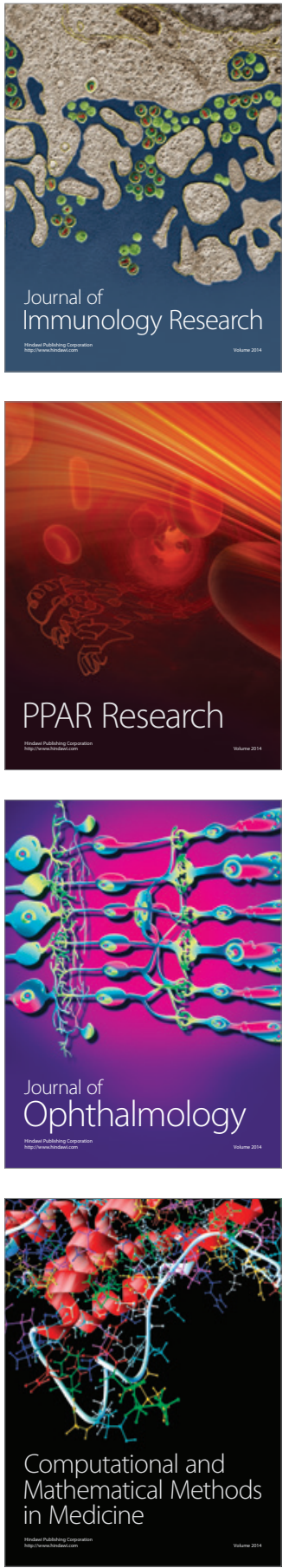

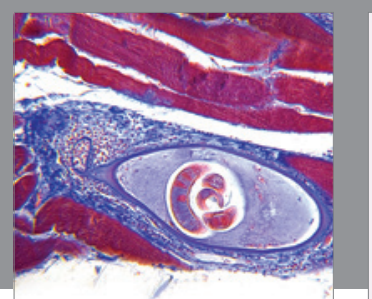

Gastroenterology Research and Practice

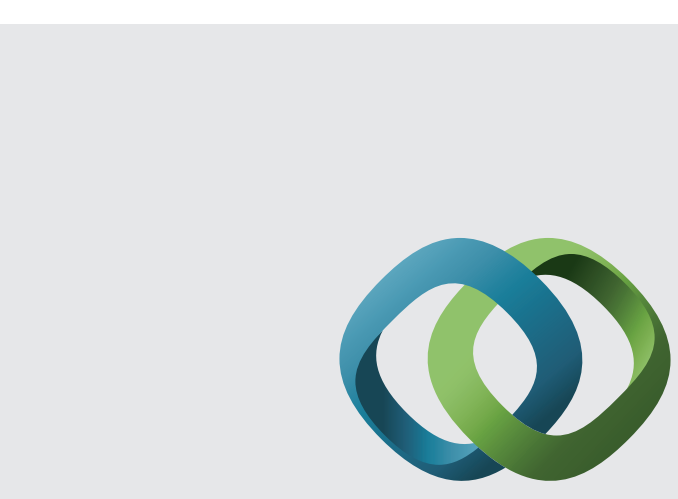

\section{Hindawi}

Submit your manuscripts at

http://www.hindawi.com
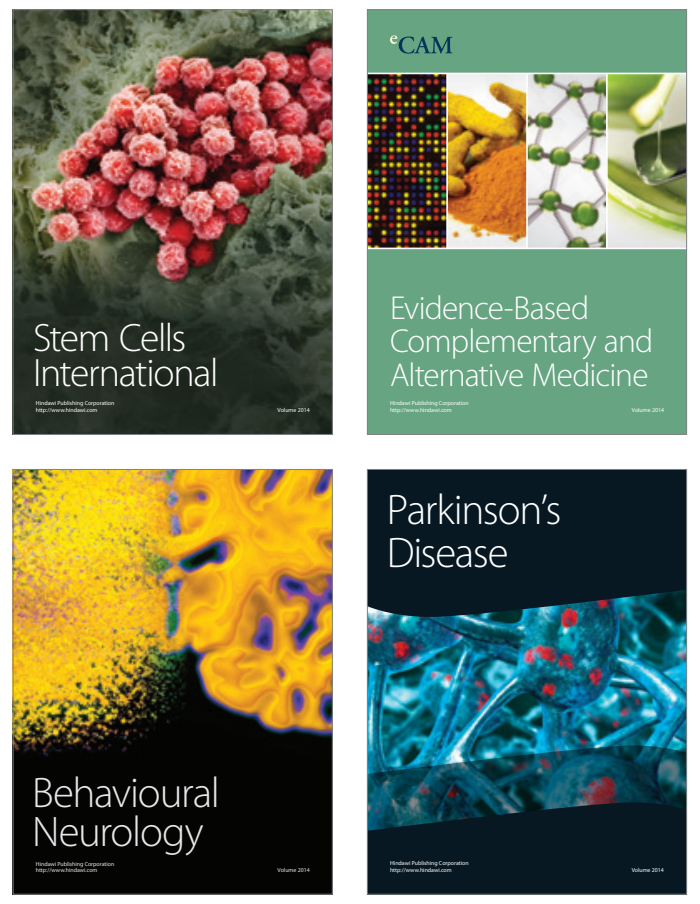
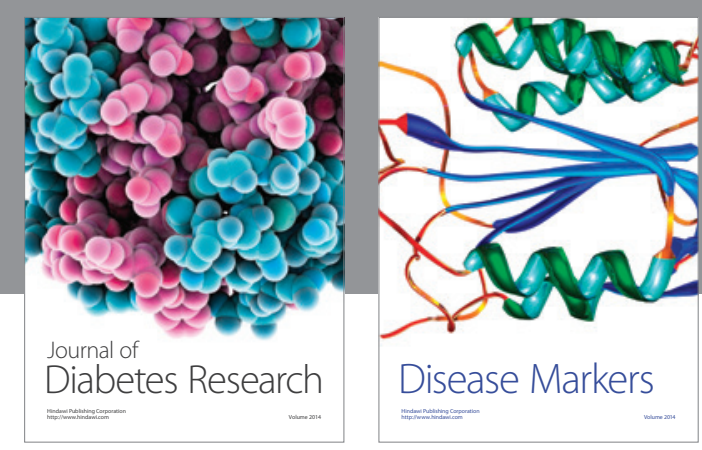

Disease Markers
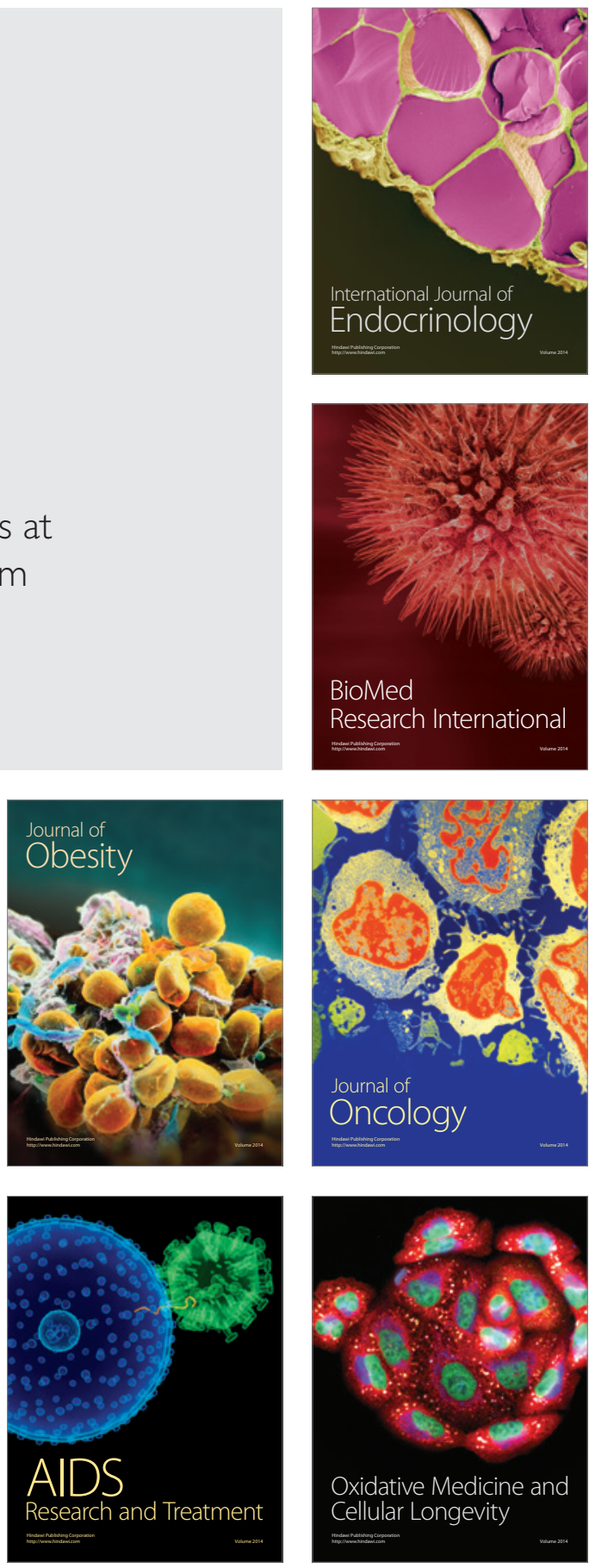